\title{
Topological quantum phase transitions in the spin-singlet superconductor with Rashba and Dresselhaus (110) spin-orbit couplings
}

\author{
Jiabin You ${ }^{1, *}$ A. H. Chan ${ }^{2, \text { ก C. H. Oh }}{ }^{1,2, \text { 用 and Vlatko Vedral }}{ }^{1,2,3,8}$ \\ ${ }^{1}$ Centre for Quantum Technologies, National University of Singapore, 117543, Singapore \\ ${ }^{2}$ Department of Physics, National University of Singapore, 117542, Singapore \\ ${ }^{3}$ Department of Physics, University of Oxford, Clarendon Laboratory, Oxford, OX1 3PU, United Kingdom
}

(Dated: May 30, 2022)

\begin{abstract}
We examine the topological properties of a spin-singlet superconductor with Rashba and Dresselhaus (110) spin-orbit couplings. We demonstrate that there are several topological invariants in the Bogoliubov-de Gennes (BdG) Hamiltonian by symmetry analysis. We use the Pfaffian invariant $\mathcal{P}$ for the particle-hole symmetry to demonstrate all the possible phase diagrams of the BdG Hamiltonian. We find that the edge spectrum is either Dirac cone or flat band which supports the emergence of the Majorana fermion in this system. For the Majorana flat bands, a higher symmetric BdG Hamiltonian is needed to make them topologically stable. The Pfaffian invariant $\mathcal{P}\left(k_{y}\right)$ and the winding number $\mathcal{W}\left(k_{y}\right)$ are used in determining the location of the Majorana flat bands.
\end{abstract}

\section{INTRODUCTION}

A topological superconductor has topologically protected gapless edge states, some of which can host Majorana fermions 110. There are two kinds of edge states in the topological superconductor. One is a Dirac cone, the other is a flat band, namely, dispersionless zeroenergy state 11 15. The Dirac cone can be found in the fully gapped topological superconductors when the Chern number of the occupied bands is nonzero. However, the flat band can appear in the fully gapped topological superconductors as well as the gapless topological superconductors which, apart from the particle-hole symmetry, have some extra symmetries in the Hamiltonian. Such flat bands are known to occur at the zigzag and bearded edge in graphene $e^{16}$, in the noncentrosymmetric superconductor $\frac{8|11| 13 \mid 14}{}$ and in other systems with topologically stable Dirac points 17 . The flat dispersion implies a peak in the density of states which has a visible experimental signature in the tunneling conductance measurements $\sqrt{18}$. The zero-bias conductance peak has been observed in recent experiments on the InSb nanowire ${ }^{[19 / 20}$ and $\mathrm{Cu}_{x} \mathrm{Bi}_{2} \mathrm{Se}_{3}{ }^{21 / 22}$ and might be due to the flat bands.

In this paper, we shall investigate the spin-singlet superconductor with the Rashba and Dresselhaus (110) spin-orbit couplings. Especially, we focus on the Hamiltonian with spin-orbit coupling of Dresselhaus (110) type which is a gapless topological system containing two kinds of edge states mentioned above. We also apply a magnetic field to the superconductor and consider the Zeeman effect. The Bogoliubov-de Gennes (BdG) Hamiltonian of the superconductor is generically particle-hole symmetric so that we can associate a Pfaffian invariant $\mathcal{P}$ with it as a topological invariant of the system. The Pfaffian invariant $\mathcal{P}$ can be used in distinguishing the topologically nontrivial phase from the trivial one. The nontrivial topological phase in this BdG Hamiltonian is a Majorana type which can be exploited for implementing fault-tolerant topological quantum computing schemes that are inherently decoherence-free 2324 . All the possible phase diagrams in the BdG Hamiltonian are demonstrated in our study. Furthermore, we find that the BdG Hamiltonian can have partial particle-hole symmetry and chiral symmetry which can be used to define the one dimensional Pfaffian invariant $\mathcal{P}\left(k_{y}\right)$ and the winding number $\mathcal{W}\left(k_{y}\right)$. The Pfaffian invariant $\mathcal{P}\left(k_{y}\right)$ and the winding number $\mathcal{W}\left(k_{y}\right)$ can be used in determining the location of the zero-energy Majorana flat band. We show that only when the system has these extra symmetries, the Majorana flat band will emerge so that a zero-bias conductance peak in the tunneling conductance measurements can be observed 19 22.

The paper is organized as follows. The BdG Hamiltonian for the spin-singlet superconductor with the Rashba and Dresselhaus (110) spin-orbit couplings is given in Sec. III. In Sec. III] we discuss the symmetries of the BdG Hamiltonian and the topological invariants associated with these symmetries are given in Sec. IV. All the possible phase diagrams of the BdG Hamiltonian are discussed in Sec. $\mathrm{V}$ whilst the edge spectra of the BdG Hamiltonian are demonstrated in Sec. VI Finally, we give a brief summary in Sec. VII.

\section{MODEL}

We model the spin-singlet superconductor on a square lattice. The kinetic energy is

$$
H_{\text {kin }}=-t \sum_{i s} \sum_{\hat{\nu}=\hat{x}, \hat{y}}\left(c_{i+\hat{\nu} s}^{\dagger} c_{i s}+c_{i-\hat{\nu} s}^{\dagger} c_{i s}\right)-\mu \sum_{i s} c_{i s}^{\dagger} c_{i s},
$$

where $c_{i s}^{\dagger}\left(c_{i s}\right)$ is the creation (annihilation) operator of the electron with spin $s=(\uparrow, \downarrow)$ at site $i=\left(i_{x}, i_{y}\right), \hat{x}(\hat{y})$ is the unit vector in the $x(y)$ direction, and $t$ is the hopping amplitude and $\mu$ is the chemical potential. For the spin-singlet superconductor, we study the $s$-wave and $d$ wave pairings in this work. The $s$-wave superconducting 
term in the square lattice is

$$
H_{s}=\sum_{i}\left[\left(\Delta_{s_{1}}+i \Delta_{s_{2}}\right) c_{i \uparrow}^{\dagger} c_{i \downarrow}^{\dagger}+\text { H.c. }\right] .
$$

Similarly, the $d$-wave superconducting term is

$$
\begin{aligned}
H_{d}= & \sum_{i}\left[\frac { \Delta _ { d _ { 1 } } } { 2 } \left(c_{i-\hat{y} \uparrow}^{\dagger} c_{i \downarrow}^{\dagger}+c_{i+\hat{y} \uparrow}^{\dagger} c_{i \downarrow}^{\dagger}\right.\right. \\
& \left.-c_{i-\hat{x} \uparrow}^{\dagger} c_{i \downarrow}^{\dagger}-c_{i+\hat{x} \uparrow}^{\dagger} c_{i \downarrow}^{\dagger}\right) \\
& +i \frac{\Delta_{d_{2}}}{4}\left(c_{i-\hat{x}+\hat{y} \uparrow}^{\dagger} c_{i \downarrow}^{\dagger}+c_{i+\hat{x}-\hat{y} \uparrow}^{\dagger} c_{i \downarrow}^{\dagger}\right. \\
& \left.\left.-c_{i+\hat{x}+\hat{y} \uparrow}^{\dagger} c_{i \downarrow}^{\dagger}-c_{i-\hat{x}-\hat{y} \uparrow}^{\dagger} c_{i \downarrow}^{\dagger}\right)+ \text { H.c. }\right] .
\end{aligned}
$$

We assume that all the superconducting parameters $\Delta_{s_{1}}, \Delta_{s_{2}}, \Delta_{d_{1}}$ and $\Delta_{d_{2}}$ are uniform in the whole superconductor. The spin-orbit couplings can arise from structure inversion asymmetry of a confinement potential (e.g., external electric field) or bulk inversion asymmetry of the underlying crystal (e.g., the zinc blende structure 25 . These two kinds of asymmetries lead to the well-known Rashba and Dresselhaus spin-orbit couplings. The Rashba spin-orbit coupling in the square lattice is of the form

$$
\begin{aligned}
H_{\mathrm{R}}= & -\frac{\alpha}{2} \sum_{i}\left[\left(c_{i-\hat{x} \downarrow}^{\dagger} c_{i \uparrow}-c_{i+\hat{x} \downarrow}^{\dagger} c_{i \uparrow}\right)\right. \\
& \left.+i\left(c_{i-\hat{y} \downarrow}^{\dagger} c_{i \uparrow}-c_{i+\hat{y} \downarrow}^{\dagger} c_{i \uparrow}\right)+\text { H.c. }\right],
\end{aligned}
$$

where $\alpha$ is the coupling strength of the Rashba spin-orbit coupling. The Dresselhaus (110) spin-orbit coupling is formulated as

$$
H_{\mathrm{D}}^{110}=-i \frac{\beta}{2} \sum_{i s s^{\prime}}\left(\tau_{z}\right)_{s s^{\prime}}\left(c_{i-\hat{x} s}^{\dagger} c_{i s^{\prime}}-c_{i+\hat{x} s}^{\dagger} c_{i s^{\prime}}\right),
$$

where $\beta$ is the coupling strength for the Dresselhaus (110) spin-orbit coupling. (110) are the common-used Miller index. We also apply an arbitrary magnetic field to the superconductor. By neglecting the orbital effect of the magnetic field, we consider the Zeeman effect as

$$
H_{\mathrm{Z}}=\sum_{i s s^{\prime}}(\mathbf{V} \cdot \tau)_{s s^{\prime}} c_{i s}^{\dagger} c_{i s^{\prime}}
$$

where $\mathbf{V}=\frac{g \mu_{B}}{2}\left(B_{x}, B_{y}, B_{z}\right) \equiv\left(V_{x}, V_{y}, V_{z}\right)$ and $\tau=$ $\left(\tau_{x}, \tau_{y}, \tau_{z}\right)$ are Pauli matrices operating on spin space. Therefore, the spin-singlet superconductor with the Rashba and Dresselhaus (110) spin-orbit couplings in an arbitrary magnetic field is dictated by the Hamiltonian $H=H_{\text {kin }}+H_{s}+H_{d}+H_{R}+H_{\mathrm{D}}^{110}+H_{\mathrm{Z}}$. In the momentum space, the Hamiltonian is recast into $H=\frac{1}{2} \sum_{\mathbf{k}} \psi_{\mathbf{k}}^{\dagger} \mathcal{H}(\mathbf{k}) \psi_{\mathbf{k}}$ with $\psi_{\mathbf{k}}^{\dagger}=\left(c_{\mathbf{k} \uparrow}^{\dagger}, c_{\mathbf{k} \downarrow}^{\dagger}, c_{-\mathbf{k} \uparrow}, c_{-\mathbf{k} \downarrow}\right)$, where $c_{\mathbf{k} s}^{\dagger}=(1 / \sqrt{N}) \sum_{\mathbf{l}} e^{i \mathbf{k} \cdot \mathbf{l}} c_{\mathbf{l} s}^{\dagger}$. Finally, the Bogoliubovde Gennes Hamiltonian $\mathcal{H}(\mathbf{k})$ for the superconductor is

$$
\left[\begin{array}{cc}
\xi(\mathbf{k})+(\mathcal{L}(\mathbf{k})+\mathbf{V}) \cdot \tau & i \Delta(\mathbf{k}) \tau_{y} \\
-i \Delta^{*}(\mathbf{k}) \tau_{y} & -\xi(\mathbf{k})+(\mathcal{L}(\mathbf{k})-\mathbf{V}) \cdot \tau^{*}
\end{array}\right]
$$

where $\xi(\mathbf{k})=-2 t\left(\cos k_{x}+\cos k_{y}\right)-\mu, \Delta(\mathbf{k})=\left(\Delta_{s_{1}}+\right.$ $\left.i \Delta_{s_{2}}\right)+\left(\Delta_{d_{1}}\left(\cos k_{y}-\cos k_{x}\right)+i \Delta_{d_{2}} \sin k_{x} \sin k_{y}\right)$ and $\mathcal{L}(\mathbf{k})=\left(\alpha \sin k_{y},-\alpha \sin k_{x}, \beta \sin k_{x}\right)$.

\section{SYMMETRIES OF THE BDG HAMILTONIAN}

For the general BdG Hamiltonian Eq. (7), it satisfies the particle-hole symmetry

$$
\Xi^{-1} \mathcal{H}(\mathbf{k}) \Xi=-\mathcal{H}(-\mathbf{k})
$$

where $\Xi=\Lambda K, \Lambda=\sigma_{x} \otimes \tau_{0}$ and $K$ is the complex conjugation operator. We find that apart from the particlehole symmetry, the BdG Hamiltonian can satisfy some extra symmetries, namely, partial particle-hole symmetry, chiral symmetry and partial chiral symmetry when we set some parameters in the Hamiltonian Eq. (7) to 0. The particle-hole- $k_{x}$ and particle-hole- $k_{y}$ symmetries are defined as

$$
\Xi_{k_{x}}^{-1} \mathcal{H}\left(k_{x}, k_{y}\right) \Xi_{k_{x}}=-\mathcal{H}\left(-k_{x}, k_{y}\right)
$$

and

$$
\Xi_{k_{y}}^{-1} \mathcal{H}\left(k_{x}, k_{y}\right) \Xi_{k_{y}}=-\mathcal{H}\left(k_{x},-k_{y}\right),
$$

where $\Xi_{k_{x}}\left(\Xi_{k_{y}}\right)$ takes the $k_{x}\left(k_{y}\right)$ in the Hamiltonian to $-k_{x}\left(-k_{y}\right)$. The chiral symmetry is

$$
\Sigma^{-1} \mathcal{H}(\mathbf{k}) \Sigma=-\mathcal{H}(\mathbf{k}) .
$$

The chiral- $k_{x}$ and chiral- $k_{y}$ symmetries are defined as

$$
\Sigma_{k_{x}}^{-1} \mathcal{H}\left(k_{x}, k_{y}\right) \Sigma_{k_{x}}=-\mathcal{H}\left(-k_{x}, k_{y}\right)
$$

and

$$
\Sigma_{k_{y}}^{-1} \mathcal{H}\left(k_{x}, k_{y}\right) \Sigma_{k_{y}}=-\mathcal{H}\left(k_{x},-k_{y}\right),
$$

where $\Sigma_{k_{x}}\left(\Sigma_{k_{y}}\right)$ takes the $k_{x}\left(k_{y}\right)$ in the Hamiltonian to $-k_{x}\left(-k_{y}\right)$.

We are interested in the BdG Hamiltonian which has one or more extra symmetries. In the following sections, we would like to consider these kinds of BdG Hamiltonian as listed in Tab. (I). The spin-singlet superconductor with Rashba spin-orbit coupling has been extensively investigated in the reference ${ }^{3}$. Here we only consider the general $d_{x^{2}-y^{2}}+i d_{x y}+s$ pairing case (a) for the superconductor with Rashba spin-orbit coupling. We shall focus on the superconductor with Dresselhaus (110) spin-orbit coupling as shown in the case (b)-(g) in Tab. (I)

\section{TOPOLOGICAL INVARIANTS OF THE BDG HAMILTONIAN}

For the fully gapped Hamiltonian, we can always define Chern number as a topological invariant of the system given by

$$
\mathcal{C}=\frac{1}{2 \pi} \int_{F B Z} d^{2} \mathbf{k} \mathcal{F}^{-}(\mathbf{k})
$$




\begin{tabular}{|c|c|c|c|c|c|}
\hline case & spin-orbit coupling & magnetic field & pairing symmetry & Hamiltonian symmetry & topological invariant \\
\hline (a) & $\alpha$ & $V_{z}$ & $\Delta_{s_{1}}, \Delta_{d_{1}}, \Delta_{d_{2}}$ & $\Xi, \Sigma_{k_{x}}$ & $\mathcal{P}, \mathcal{W}$ \\
\hline (b) & \multirow{6}{*}{$\beta$} & \multirow{6}{*}{$V_{x}, V_{y}$} & $\Delta_{s_{1}}$ & $\Xi, \Xi_{k_{x}}, \Sigma, \Sigma_{k_{y}}$ & $\mathcal{P}, \mathcal{P}\left(k_{y}\right), \mathcal{W}, \mathcal{W}\left(k_{y}\right)$ \\
\hline (c) & & & $\Delta_{s_{1}}, \Delta_{s_{2}}$ & $\Xi, \Xi_{k_{x}}$ & $\mathcal{P}, \mathcal{P}\left(k_{y}\right)$ \\
\hline (d) & & & $\Delta_{d_{1}}$ & $\Xi, \Xi_{k_{x}}, \Sigma, \Sigma_{k_{y}}$ & $\mathcal{P}, \mathcal{P}\left(k_{y}\right), \mathcal{W}, \mathcal{W}\left(k_{y}\right)$ \\
\hline (e) & & & $\Delta_{d_{1}}, \Delta_{d_{2}}$ & $\Xi, \Sigma_{k_{y}}$ & $\mathcal{P}, \mathcal{W}$ \\
\hline (f) & & & $\Delta_{s_{1}}, \Delta_{d_{1}}$ & $\Xi, \Xi_{k_{x}}, \Sigma$ & $\mathcal{P}, \mathcal{P}\left(k_{y}\right), \mathcal{W}\left(k_{y}\right)$ \\
\hline (g) & & & $\Delta_{s_{1}}, \Delta_{d_{1}}, \Delta_{d_{2}}$ & $\Xi, \Sigma_{k_{y}}$ & $\mathcal{P}, \mathcal{W}$ \\
\hline
\end{tabular}

TABLE I. The BdG Hamiltonian with extra symmetries, namely, the particle-hole symmetry and the particle-hole- $k_{x}$ symmetry, $\Xi=\Xi_{k_{x}}=\sigma_{x} K$, the chiral symmetry and the chiral- $k_{y}$ symmetry, $\Sigma=\Sigma_{k_{y}}=i \sigma_{y} \tau_{x}$ and the chiral- $k_{x}$ symmetry, $\Sigma_{k_{x}}=i \sigma_{y} \tau_{z}$. The topological invariants for these extra symmetries are also shown in the last column.

Here $\mathcal{F}^{-}(\mathbf{k})=\epsilon^{i j} \partial_{k_{i}} A_{j}^{-}(\mathbf{k})$ is the strength of the gauge field $\mathbf{A}^{-}(\mathbf{k})=i \sum_{n<0}\left\langle\psi_{n}(\mathbf{k}) \mid \nabla \psi_{n}(\mathbf{k})\right\rangle$, where $\psi_{n}(\mathbf{k})$ is the eigenstate of the Hamiltonian. The integral is carried out in the first Brillouin zone (FBZ) and the summation is carried out for the occupied states. If the Hamiltonian has some extra symmetries, more topological invariants can be introduced into the system.

We first consider the particle-hole symmetry Eq. (8) which can be reduced to $\Lambda \mathcal{H}(\mathbf{k}) \Lambda=-\mathcal{H}^{*}(-\mathbf{k})$. We find that under this symmetry $\mathcal{H}(\mathbf{K}) \Lambda$ is an antisymmetric matrix with $(\mathcal{H}(\mathbf{K}) \Lambda)^{T}=-\mathcal{H}(\mathbf{K}) \Lambda$, where $\mathbf{K}$ is the particle-hole symmetric momenta satisfying $\mathbf{K}=-\mathbf{K}+\mathbf{G}$ and $\mathbf{G}$ is the reciprocal lattice vector of the square lattice. With this property, we can define the Pfaffian invariant for the particle-hole symmetric Hamiltonian as 26

$$
\mathcal{P}=\operatorname{sgn}\left\{\frac{\operatorname{Pf}\left[\mathcal{H}\left(\mathbf{K}_{\mathbf{1}}\right) \Lambda\right] \operatorname{Pf}\left[\mathcal{H}\left(\mathbf{K}_{\mathbf{4}}\right) \Lambda\right]}{\operatorname{Pf}\left[\mathcal{H}\left(\mathbf{K}_{\mathbf{2}}\right) \Lambda\right] \operatorname{Pf}\left[\mathcal{H}\left(\mathbf{K}_{\mathbf{3}}\right) \Lambda\right]}\right\}
$$

where $\mathbf{K}_{\mathbf{1}}=(0,0), \mathbf{K}_{\mathbf{2}}=(\pi, 0), \mathbf{K}_{\mathbf{3}}=(0, \pi)$ and $\mathbf{K}_{\mathbf{4}}=(\pi, \pi)$ are the four particle-hole symmetric momenta in the first Brillouin zone of the square lattice. Here we shall show that the Pfaffian invariant $\mathcal{P}$ is the parity of the Chern number $\mathcal{C}, \mathcal{P}=$ $(-1)^{\mathcal{C}}$. For the $2 n \times 2 n$ antisymmetric matrix $\mathcal{H}(\mathbf{K}) \Lambda$, we have $\operatorname{Pf}[\mathcal{H}(\mathbf{K}) \Lambda]^{*}=(-1)^{n} \operatorname{Pf}[\mathcal{H}(\mathbf{K}) \Lambda]$. Therefore, $\left(i^{n} \operatorname{Pf}[\mathcal{H}(\mathbf{K}) \Lambda]\right)^{*}=i^{n} \operatorname{Pf}[\mathcal{H}(\mathbf{K}) \Lambda]$ is real so that we can define a quantity $S[\mathcal{H}(\mathbf{K})]=\operatorname{sgn}\left\{i^{n} \operatorname{Pf}[\mathcal{H}(\mathbf{K}) \Lambda]\right\}$ for any particle-hole symmetric Hamiltonian. Suppose $\mathcal{H}(\mathbf{K})$ is diagonalized by the transformation $\mathcal{H}(\mathbf{K})=$ $U(\mathbf{K}) D(\mathbf{K}) U^{\dagger}(\mathbf{K})$, where $D(\mathbf{K})$ is a diagonal matrix of eigenvalues $\operatorname{diag}\left\{E_{n}(\mathbf{K}), \cdots, E_{1}(\mathbf{K}),-E_{1}(\mathbf{K}), \cdots\right.$ $\left.\cdot,-E_{n}(\mathbf{K})\right\}$ and the columns of the unitary matrix $U(\mathbf{K})$ are the eigenvectors of $\mathcal{H}(\mathbf{K})$. The eigenvectors for positive eigenvalues in $U(\mathbf{K})$ are chosen to be related to the eigenvectors for negative eigenvalues by particle-hole symmetry. With this convention, we find that $U^{\dagger} \Lambda=$ $\Gamma U^{T}$, where $\Gamma=\sigma_{x} \tau_{x}$. Therefore, $S[\mathcal{H}(\mathbf{K})]$ can be further reduced to

$$
\begin{aligned}
S[\mathcal{H}(\mathbf{K})] & =\operatorname{sgn}\left\{i^{n} \operatorname{Pf}[\mathcal{H}(\mathbf{K}) \Lambda]\right\}, \\
& =\operatorname{sgn}\left\{i^{n} \operatorname{Pf}\left[U(\mathbf{K}) D(\mathbf{K}) U^{\dagger}(\mathbf{K}) \Lambda\right]\right\}, \\
& =\operatorname{sgn}\left\{i^{n} \operatorname{Pf}\left[U(\mathbf{K}) D(\mathbf{K}) \Gamma U^{T}(\mathbf{K})\right]\right\}, \\
& =\operatorname{sgn}\left\{i^{n} \operatorname{det} U(\mathbf{K}) \operatorname{Pf}[D(\mathbf{K}) \Gamma]\right\} .
\end{aligned}
$$

Since $\operatorname{Pf}[D(\mathbf{K}) \Gamma]=\prod_{n>0} E_{n}(\mathbf{K})>0$ and $|\operatorname{det} U(\mathbf{K})|=$
1 , we arrive at

$$
S[\mathcal{H}(\mathbf{K})]=i^{n} \operatorname{det} U(\mathbf{K}) .
$$

Note that $\mathbf{A}(\mathbf{k})=i \sum_{n}\left\langle\psi_{n}(\mathbf{k}) \mid \nabla \psi_{n}(\mathbf{k})\right\rangle$ is a total derivative ${ }^{3} \mathbf{A}(\mathbf{k})=i \nabla \ln [\operatorname{det} U(\mathbf{k})]$. Therefore, consider a pair of particle-hole symmetric momenta $\mathbf{K}_{\mathbf{1}}$ and $\mathbf{K}_{\mathbf{2}}$, we find that

$$
\frac{\operatorname{det} U\left(\mathbf{K}_{\mathbf{2}}\right)}{\operatorname{det} U\left(\mathbf{K}_{\mathbf{1}}\right)}=e^{-i S_{1,2}},
$$

where $S_{1,2}=\int_{\mathbf{K}_{1}}^{\mathbf{K}_{2}} \mathbf{A}(\mathbf{k}) \cdot d \mathbf{k}$ and the line integral runs from $\mathbf{K}_{\mathbf{1}}$ to $\mathbf{K}_{\mathbf{2}}$. Since $\mathbf{A}^{+}(\mathbf{k})=$ $i \sum_{n>0}\left\langle\psi_{n}(\mathbf{k}) \mid \nabla \psi_{n}(\mathbf{k})\right\rangle=\mathbf{A}^{-}(-\mathbf{k})$, we find that $S_{1,2}=$ $\int_{\gamma_{1}} \mathbf{A}^{-}(\mathbf{k}) \cdot d \mathbf{k}$, where $\gamma_{1}$ is the line from $(-\pi, 0)$ to $(\pi, 0)$. Similarly,

$$
\frac{\operatorname{det} U\left(\mathbf{K}_{\mathbf{4}}\right)}{\operatorname{det} U\left(\mathbf{K}_{\mathbf{3}}\right)}=e^{-i S_{3,4}},
$$

where $S_{3,4}=\int_{\gamma_{2}} \mathbf{A}^{-}(\mathbf{k}) \cdot d \mathbf{k}$ and $\gamma_{2}$ is the line from $(-\pi, \pi)$ to $(\pi, \pi)$. Therefore,

$$
\frac{\operatorname{det} U\left(\mathbf{K}_{\mathbf{1}}\right) \operatorname{det} U\left(\mathbf{K}_{\mathbf{4}}\right)}{\operatorname{det} U\left(\mathbf{K}_{\mathbf{2}}\right) \operatorname{det} U\left(\mathbf{K}_{\mathbf{3}}\right)}=e^{i S_{\gamma}},
$$

where $S_{\gamma}=\oint_{\gamma} \mathbf{A}^{-}(\mathbf{k}) \cdot d \mathbf{k}$ and $\gamma$ is the directed line surrounding the upper half Brillouin zone (UHBZ) in the counter clockwise direction. Since $\mathcal{F}^{-}(\mathbf{k})=\mathcal{F}^{-}(-\mathbf{k})$, we have

$$
\begin{aligned}
S_{\gamma} & =\oint_{\gamma} \mathbf{A}^{-}(\mathbf{k}) \cdot d \mathbf{k}, \\
& =\int_{U H B Z} d^{2} \mathbf{k} \mathcal{F}^{-}(\mathbf{k}), \\
& =\frac{1}{2} \int_{F B Z} d^{2} \mathbf{k} \mathcal{F}^{-}(\mathbf{k}), \\
& =\pi \mathcal{C} .
\end{aligned}
$$

Finally, we obtain that

$$
\begin{aligned}
\mathcal{P} & =\operatorname{sgn}\left\{\frac{\operatorname{Pf}\left[\mathcal{H}\left(\mathbf{K}_{\mathbf{1}}\right) \Lambda\right] \operatorname{Pf}\left[\mathcal{H}\left(\mathbf{K}_{\mathbf{4}}\right) \Lambda\right]}{\operatorname{Pf}\left[\mathcal{H}\left(\mathbf{K}_{\mathbf{2}}\right) \Lambda\right] \operatorname{Pf}\left[\mathcal{H}\left(\mathbf{K}_{\mathbf{3}}\right) \Lambda\right]}\right\}, \\
& =\frac{\operatorname{det} U\left(\mathbf{K}_{\mathbf{1}}\right) \operatorname{det} U\left(\mathbf{K}_{\mathbf{4}}\right)}{\operatorname{det} U\left(\mathbf{K}_{\mathbf{2}}\right) \operatorname{det} U\left(\mathbf{K}_{\mathbf{3}}\right)}, \\
& =(-1)^{\mathcal{C}} .
\end{aligned}
$$


Therefore, the Pfaffian invariant $\mathcal{P}$ is the parity of the Chern number.

Similarly, if the Hamiltonian has partial particle-hole symmetry, for example, the particle-hole- $k_{x}$ symmetry Eq. (9), then we can treat $k_{y}$ as a parameter and define the Pfaffian invariant $\mathcal{P}\left(k_{y}\right)$ to identify the location of the edge states in the edge Brillouin zone $e^{7 / 15}$,

$$
\mathcal{P}\left(k_{y}\right)=\operatorname{sgn}\left\{\frac{\operatorname{Pf}\left[\mathcal{H}\left(\pi, k_{y}\right) \Lambda\right]}{\operatorname{Pf}\left[\mathcal{H}\left(0, k_{y}\right) \Lambda\right]}\right\},
$$

where $k_{x}=0$ and $k_{x}=\pi$ are the two particle-hole symmetric momenta in the edge Brillouin zone. Similar to the Eq. 22, we can get an expression of $\mathcal{P}\left(k_{y}\right)$ in terms of the line integral of the vector potential $A_{x}^{-}(\mathbf{k})$ as

$$
\mathcal{P}\left(k_{y}\right)=(-1)^{\frac{1}{\pi} \int_{-\pi}^{\pi} d k_{x} A_{x}^{-}(\mathbf{k})} .
$$

If the Hamiltonian has chiral symmetry Eq. [11), then the winding number can be introduced as a topological invariant of the system. The $\Sigma$ appearing in this paper can be diagonalized as $\Sigma=T D T^{\dagger}$ with $T T^{\dagger}=1$ and $D=\operatorname{diag}\{i, i,-i,-i\}$. The Hamiltonian $\mathcal{H}(\mathbf{k})$ is then simultaneously off-diagonalized as $\mathcal{H}(\mathbf{k})=T Q(\mathbf{k}) T^{\dagger}$, where $Q(\mathbf{k})$ is of the form $\left(\begin{array}{cc}0 & q(\mathbf{k}) \\ q^{\dagger}(\mathbf{k}) & 0\end{array}\right)$. We can thus define the winding number as

$$
\begin{aligned}
\mathcal{W}\left(k_{y}\right) & =-\frac{1}{4 \pi} \int_{-\pi}^{\pi} d k_{x} \operatorname{tr}\left[\Sigma \mathcal{H}^{-1}(\mathbf{k}) \partial_{k_{x}} \mathcal{H}(\mathbf{k})\right] \\
& =-\frac{1}{4 \pi} \int_{-\pi}^{\pi} d k_{x} \operatorname{tr}\left[D Q^{-1}(\mathbf{k}) \partial_{k_{x}} Q(\mathbf{k})\right] \\
& =\frac{i}{4 \pi} \int_{-\pi}^{\pi} d k_{x} \operatorname{tr}\left[q^{-1}(\mathbf{k}) \partial_{k_{x}} q(\mathbf{k})-q^{\dagger-1}(\mathbf{k}) \partial_{k_{x}} q^{\dagger}(\mathbf{k})\right] \\
& =-\frac{1}{2 \pi} \operatorname{Im} \int_{-\pi}^{\pi} d k_{x} \operatorname{tr}\left[q^{-1}(\mathbf{k}) \partial_{k_{x}} q(\mathbf{k})\right] .
\end{aligned}
$$

Here we shall show that $\int_{-\pi}^{\pi} d k_{x} \operatorname{tr}\left[q^{-1}(\mathbf{k}) \partial_{k_{x}} q(\mathbf{k})\right]$ is pure imaginary. It is easy to see $\operatorname{tr}\left[q^{-1} \partial_{k_{x}} q\right]^{*}=$ $-\operatorname{tr}\left[q^{\dagger} \partial_{k_{x}} q^{\dagger-1}\right]$. From the eigen equation of $Q(\mathbf{k})$, we find that $q q^{\dagger}\left|\psi_{n}\right\rangle=E_{n}^{2}\left|\psi_{n}\right\rangle$ which leads to the identity $q q^{\dagger} \Psi=\Psi \Pi$, where $\Pi=\operatorname{diag}\left\{E_{1}^{2}, E_{2}^{2}\right\}$ and the unitary matrix $\Psi=\left(\left|\psi_{1}\right\rangle,\left|\psi_{2}\right\rangle\right)$. Therefore, we obtain $\operatorname{tr}\left[q^{\dagger} \partial_{k_{x}} q^{\dagger-1}\right]=\operatorname{tr}\left[q^{-1} \partial_{k_{x}} q\right]+\operatorname{tr}\left[\Pi \partial_{k_{x}} \Pi^{-1}\right]$ and accordingly,

$$
\begin{gathered}
\int_{-\pi}^{\pi} d k_{x} \operatorname{tr}\left[q^{-1} \partial_{k_{x}} q\right]^{*}=-\int_{-\pi}^{\pi} d k_{x} \operatorname{tr}\left[q^{-1} \partial_{k_{x}} q\right] \quad \begin{array}{l}
\text { nontrivial topological phase. The exp } \\
\text { the Pfaffian invariant Eq. } 22 \text { for the }
\end{array} \\
-\int_{-\pi}^{\pi} d k_{x} \operatorname{tr}\left[\Pi \partial_{k_{x}} \Pi^{-1}\right] . \\
\mathcal{P}=\operatorname{sgn}\left\{\frac{\left[(\mu+4 t)^{2}+\left(\Delta_{s_{1}}^{2}+\Delta_{s_{2}}^{2}\right)-V^{2}\right]\left[(\mu-4 t)^{2}+\left(\Delta_{s_{1}}^{2}+\Delta_{s_{2}}^{2}\right)-V^{2}\right]}{\left[\mu^{2}+\left(\Delta_{s_{1}}+2 \Delta_{d_{1}}\right)^{2}+\Delta_{s_{2}}^{2}-V^{2}\right]\left[\mu^{2}+\left(\Delta_{s_{1}}-2 \Delta_{d_{1}}\right)^{2}+\Delta_{s_{2}}^{2}-V^{2}\right]}\right\} .
\end{gathered}
$$

Due to the periodic boundary condition, we have $E_{n}\left(k_{x}=-\pi, k_{y}\right)=E_{n}\left(k_{x}=\pi, k_{y}\right)$ so that

$$
\int_{-\pi}^{\pi} d k_{x} \operatorname{tr}\left[\Pi \partial_{k_{x}} \Pi^{-1}\right]=-2 \sum_{n=1}^{2} \int_{-\pi}^{\pi} d k_{x} \partial_{k_{x}} \ln E_{n}(\mathbf{k})=0
$$

Thus $\int_{-\pi}^{\pi} d k_{x} \operatorname{tr}\left[q^{-1} \partial_{k_{x}} q\right]^{*}=-\int_{-\pi}^{\pi} d k_{x} \operatorname{tr}\left[q^{-1} \partial_{k_{x}} q\right]$ is pure imaginary. Finally, the winding number for the chiral symmetry Eq. 111 is obtained,

$$
\mathcal{W}\left(k_{y}\right)=-\frac{1}{2 \pi i} \int_{-\pi}^{\pi} d k_{x} \operatorname{tr}\left[q^{-1}(\mathbf{k}) \partial_{k_{x}} q(\mathbf{k})\right] .
$$

When the Hamiltonian has partial particle-hole symmetry and chiral symmetry simultaneously, we can find a relation between the Pfaffian invariant $\mathcal{P}\left(k_{y}\right)$ and the winding number $\mathcal{W}\left(k_{y}\right)$. According to the reference ${ }^{3}$, $\frac{1}{\pi} \int_{-\pi}^{\pi} A_{x}^{-}(\mathbf{k})=\frac{1}{2 \pi i} \int_{-\pi}^{\pi} \operatorname{tr}\left[q(\mathbf{k})^{-1} \partial_{k_{x}} q(\mathbf{k})\right]+2 N$, where $N$ is an integer. Substitute this into Eq. (24), we get that $\mathcal{P}\left(k_{y}\right)=(-1)^{\mathcal{W}\left(k_{y}\right)}$. Therefore, the Pfaffian invariant $\mathcal{P}\left(k_{y}\right)$ is the parity of the winding number $\mathcal{W}\left(k_{y}\right)$.

If the Hamiltonian has partial chiral symmetry, for example, the chiral- $k_{y}$ symmetry Eq. 113, then we can only define the winding number $\mathcal{W}\left(k_{y}\right)$ at $k_{y}=0$ and $k_{y}=\pi$. Consequently, we can associate a topological invariant $\mathcal{W}$ with the chiral- $k_{y}$ symmetry as ${ }^{3}$

$$
\mathcal{W}=(-1)^{\mathcal{W}(0)-\mathcal{W}(\pi)} .
$$

The topological invariant $\mathcal{W}$ is also the parity of the Chern number, $\mathcal{W}=(-1)^{\mathcal{C}}$. Therefore, the Pfaffian invariant $\mathcal{P}$ for the particle-hole symmetry is equivalent to the topological invariant $\mathcal{W}$ for the partial chiral symmetry.

\section{PHASE DIAGRAMS OF THE BDG HAMILTONIAN}

In contrast to the even number of Majorana bound states in the trivial topological phase, the number of Majorana bound states is odd in the nontrivial topological phase. The Pfaffian invariant $\mathcal{P}$ is in fact the parity of the number of Majorana bound states. Therefore, we can use the Pfaffian invariant $\mathcal{P}$ to investigate the topological quantum phase transitions in the BdG Hamiltonian Eq. (7). The phase diagrams are shown in Fig. (1). Our interest is in the red region where the Pfaffian invariant $\mathcal{P}=-1$ which means that the system has an odd number of Majorana bound states at the edge and is thus in the nontrivial topological phase. The explicit expression of the Pfaffian invariant Eq. 22 for the general case of the 
Therefore, the phase diagram is divided by the following four parabolas in the plane of $V^{2} \sim \mu$ : (i) $V^{2}=$ $(\mu+4 t)^{2}+\left(\Delta_{s_{1}}^{2}+\Delta_{s_{2}}^{2}\right)$; (ii) $V^{2}=(\mu-4 t)^{2}+\left(\Delta_{s_{1}}^{2}+\Delta_{s_{2}}^{2}\right)$; (iii) $V^{2}=\mu^{2}+\left(\Delta_{s_{1}}+2 \Delta_{d_{1}}\right)^{2}+\Delta_{s_{2}}^{2}$ and (iv) $V^{2}=$ $\mu^{2}+\left(\Delta_{s_{1}}-2 \Delta_{d_{1}}\right)^{2}+\Delta_{s_{2}}^{2}$, where $V^{2}=V_{x}^{2}+V_{y}^{2}+V_{z}^{2}$. Notice that the Pfaffian invariant $\mathcal{P}$ has nothing to do with the spin-orbit couplings. Thus the topological phases can exist even without the spin-orbit couplings. However, the spin-orbit couplings can open a gap to render the Majorana fermion located at the edge of the system; otherwise the Majorana fermion will spread into the bulk. Now we turn to discuss all the possible phase diagrams in the BdG Hamiltonian. When $\Delta_{s_{1}} \Delta_{d_{1}}=0$, the phase diagram is only divided by the parabolas (i) and (ii) and is shown in Fig. (1p). When $\Delta_{s_{1}} \Delta_{d_{1}} \neq 0$, there are three topologically different cases in the phase diagrams as follows. Let us first define the intersection point of the parabolas (i) and (ii) as $O$, then the phase diagram where the parabolas (iii) and (iv) are both below $O$ is shown in Fig. (1b); the phase diagram where the parabolas (iii) and (iv) are on either side of $O$ is shown in Fig. (1 1 ); the phase diagram where the parabolas (iii) and (iv) are both above $O$ is shown in Fig. (1) . Furthermore, if we assume $\Delta_{s_{1}} \Delta_{d_{1}}>0$, then the phase diagram is as Fig. (1p) when $\Delta_{d_{1}}^{2}-\Delta_{s_{1}} \Delta_{d_{1}}<\Delta_{d_{1}}^{2}+\Delta_{s_{1}} \Delta_{d_{1}}<4 t^{2}$; the phase diagram is as Fig. (11) when $\Delta_{d_{1}}^{2}-\Delta_{s_{1}} \Delta_{d_{1}}<$ $4 t^{2}<\Delta_{d_{1}}^{2}+\Delta_{s_{1}} \Delta_{d_{1}}$; the phase diagram is as Fig. (1) when $4 t^{2}<\Delta_{d_{1}}^{2}-\Delta_{s_{1}} \Delta_{d_{1}}<\Delta_{d_{1}}^{2}+\Delta_{s_{1}} \Delta_{d_{1}}$. Therefore, we have exhibited all the possible phase diagrams in the BdG Hamiltonian Eq. (7) in this paper. For the pure $s$-wave and $d$-wave superconductors, the phase diagrams are topologically equivalent to Fig. (1a); for the superconductors with mixed $s$-wave and $d$-wave pairing symmetries, the phase diagrams are topologically equivalent to Fig. (1b), Fig. (1k) and Fig. (1) depending on $t$.

\section{MAJORANA BOUND STATES AT THE EDGE OF THE BDG HAMILTONIAN}

In this Section, we shall demonstrate the Majorana bound states at the edge of the spin-singlet superconductor in the different cases as listed in Tab. (I). By setting the boundary conditions of the $x$ direction to be open and the $y$ direction to be periodic, we diagonalize the Hamiltonian Eq. (7) with the cylindrical symmetry and get the edge spectrum of the Hamiltonian.

We first discuss the pure $s$-wave superconductor in case (b) and case (c). The difference between case (b) and case (c) is that the appearance of the superconducting parameter $\Delta_{s_{2}}$ in case (c) lowers the symmetry by breaking the chiral symmetry. Consequently, the number of topological invariants in case (c) is less than the one in case (b). The phase diagrams of case (b) and case (c) are topologically equivalent and are shown in Fig. (17). The edge spectra of case (b) and case (c) are exhibited in Fig. (2) and Fig. (3), respectively. We observe that the Majorana
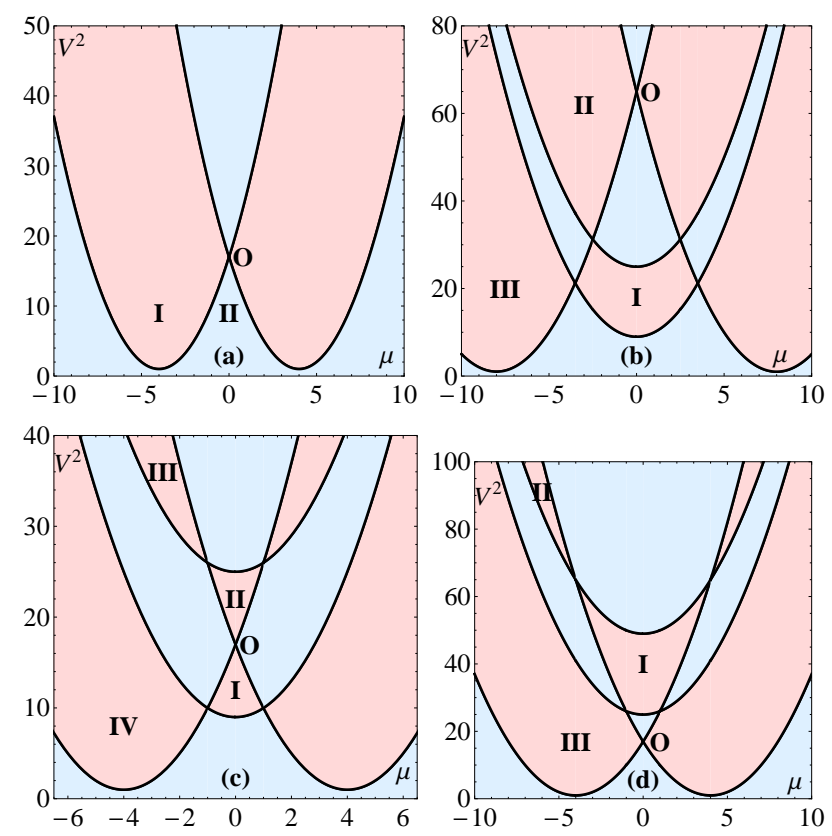

FIG. 1. (color online). The possible phase diagrams of the spin-singlet superconductor with Rashba and Dresselhaus (110) spin-orbit couplings. (a) is the phase diagram for the pure $s$-wave or $d$-wave superconductor. (b), (c) and (d) are the phase diagrams for the mixed $s$-wave and $d$-wave superconductor.

bound states in these two cases are flat bands. There are odd number of Majorana flat bands in the nontrivial topological phase. Although the edge spectra of these two cases are similar, the symmetries and the topological invariants protecting them are different as shown in Tab. (I). In case (b), we can use $\mathcal{P}$ and $\mathcal{W}$ to find the regions in the phase diagram where there exists nontrivial Majorana flat band and use $\mathcal{P}\left(k_{y}\right)$ and $\mathcal{W}\left(k_{y}\right)$ to locate the range of flat band in the edge Brillouin zone as shown in Fig. (2p) and Fig. (2d). However, in case (c), only the Pfaffian invariant $\mathcal{P}$ and $\mathcal{P}\left(k_{y}\right)$ can be used in finding the Majorana flat bands. We find that when $\mathcal{P}\left(k_{y}\right)=-1$ or $\mathcal{W}\left(k_{y}\right)$ is odd, the topologically nontrivial Majorana flat bands will emerge in the edge Brillouin zone.

For the pure $d$-wave superconductor in case (d) and (e), the phase diagram is also shown in Fig. (11). The edge spectra of the $d_{x^{2}-y^{2}}$-wave superconductor are depicted in Fig. (4). There are an odd number of Majorana flat bands in the nontrivial topological phase as shown in Fig. (4 4 ) and the location of the Majorana flat bands is consistent with the Pfaffian invariant $\mathcal{P}\left(k_{y}\right)$ and the winding number $\mathcal{W}\left(k_{y}\right)$ as shown in Fig. (4k) and (44). Note that the winding number $\mathcal{W}\left(k_{y}\right)$ can change by some even number in the same phase. However, its parity, the Pfaffian invariant $\mathcal{P}\left(k_{y}\right)$ is unchanged in the same phase since $\mathcal{P}\left(k_{y}\right)=(-1)^{\mathcal{W}\left(k_{y}\right)}$. The edge spectra of the $d_{x^{2}-y^{2}}+i d_{x y}$-wave superconductor are displayed in Fig. (5). We find that the Majorana bound states in this case get dispersed and become Dirac cones. Similarly, 
there is an odd number of Dirac cones in the nontrivial topological phase as shown in Fig. (5a). Comparing case (d) with case (e), we find that the Majorana flat bands can emerge only when the Hamiltonian has a chiral or partial particle-hole symmetry since these symmetries can provide the topological invariants $\mathcal{P}\left(k_{y}\right)$ or $\mathcal{W}\left(k_{y}\right)$ to make the Majorana flat bands stable in the edge Brillouin zone.

The superconductors with mixed $s$-wave and $d$-wave pairing symmetries are in case (a), (f) and (g). For each case, there are three different kinds of phase diagrams depending on the hopping amplitude $t$ as demonstrated in Fig. (1p)-(1 d). Although the edge spectrum becomes more complicated, there are no qualitative differences in the edge spectrum between the $d+s$-wave superconductor and the pure $d$-wave or $s$-wave superconductor. The edge spectra for the $d_{x^{2}-y^{2}}+s$-wave superconductor with Dresselhaus (110) spin-orbit coupling are shown in Fig. (6). Due to the partial particle-hole symmetry and the chiral symmetry, the edge spectra of this kind of superconductor are the Majorana flat bands protected by the Pfaffian invariant $\mathcal{P}\left(k_{y}\right)$ and the winding number $\mathcal{W}\left(k_{y}\right)$. Notice that in Fig. (6f) and Fig. (6t), the winding number $\mathcal{W}\left(k_{y}\right)$ in some range of $k_{y}$ is 2 , however, its parity namely the Pfaffian invariant $\mathcal{P}\left(k_{y}\right)$ is 1 . Therefore, the phase is trivial in this range of $k_{y}$. The edge spectra for the $d_{x^{2}-y^{2}}+i d_{x y}+s$-wave superconductor with Rashba spin-orbit coupling and Dresselhaus (110) spin-orbit coupling are shown in Fig. (7) and Fig. (8), respectively. Without the protection of the partial particle-hole symmetry or the chiral symmetry, the Majorana flat bands disappear and become Dirac cones. In the nontrivial topological phase, we find that the edge spectra have an odd number of Dirac cones.

We can define the $k_{y}$-dependent Pfaffian invariant $\mathcal{P}\left(k_{y}\right)$ or winding number $\mathcal{W}\left(k_{y}\right)$ for the partial particlehole symmetry or the chiral symmetry. At fixed $k_{y}$, there exist zero-energy states when $\mathcal{P}\left(k_{y}\right)=(-1)^{\mathcal{W}\left(k_{y}\right)}=-1$. Therefore, there are zero-energy Majorana flat bands in some range of $k_{y}$ in the edge Brillouin zone when the Hamiltonian has the partial particle-hole symmetry or the chiral symmetry. Notice that the Majorana flat band does not always situate at the edge of the system. At a fixed $k_{y}$, the bigger the gap of bulk state is, the more localized the Majorana bound state is. Let us take the

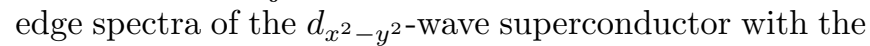
Dresselhaus (110) spin-orbit coupling in Fig. (4) as an example. The probability distributions of the quasiparticle at $k_{y}=0,1,1.3$ are shown in Fig. (9). From Fig. (4a), we see that the gap of the bulk state decreases as $k_{y}$ increases from 0 to 1.3 . At the same time, the probability distribution of the quasiparticle becomes more and more delocalized and extends into the bulk. Therefore, only the big-gap Majorana bound states in the flat bands are well-defined Majorana particles.
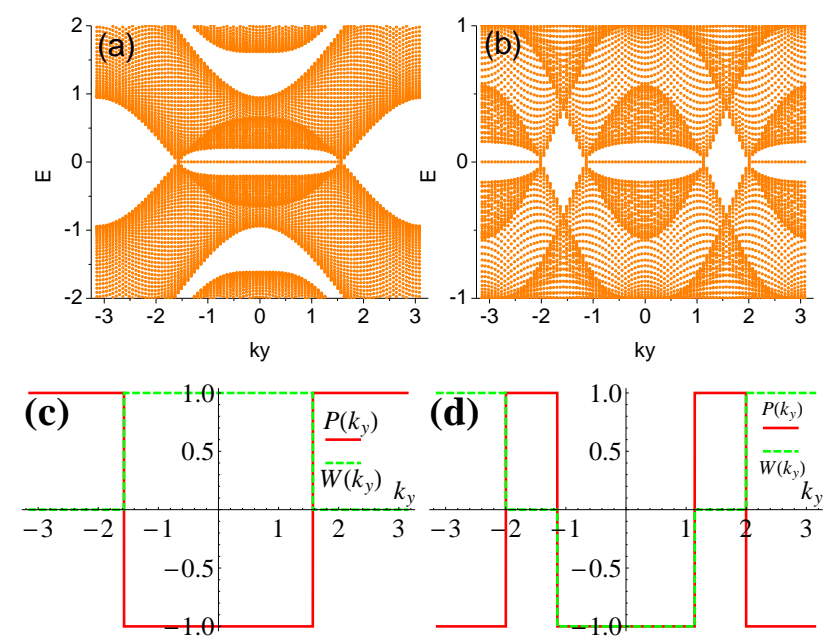

FIG. 2. (color online). (a) and (b) are the edge spectra of the $s$-wave superconductor with Dresselhaus (110) spin-orbit coupling in case (b) of Tab. (I). The open edges are at $i_{x}=0$ and $i_{x}=50, k_{y}$ denotes the momentum in the $y$ direction and $k_{y} \in(-\pi, \pi]$. The parameters are $t=1, \beta=1, \Delta_{s_{1}}=$ $1, \Delta_{s_{2}}=0$ and (a) $\mu=-4, V^{2}=5$, (b) $\mu=0, V^{2}=9$, which correspond to regions I and II in Fig. (1), respectively. (c) and (d) are the Pfaffian invariant given by Eq. (23) and winding number given by Eq. (28) for (a) and (b). This figure is cited from the reference ${ }^{8}$ for completeness.
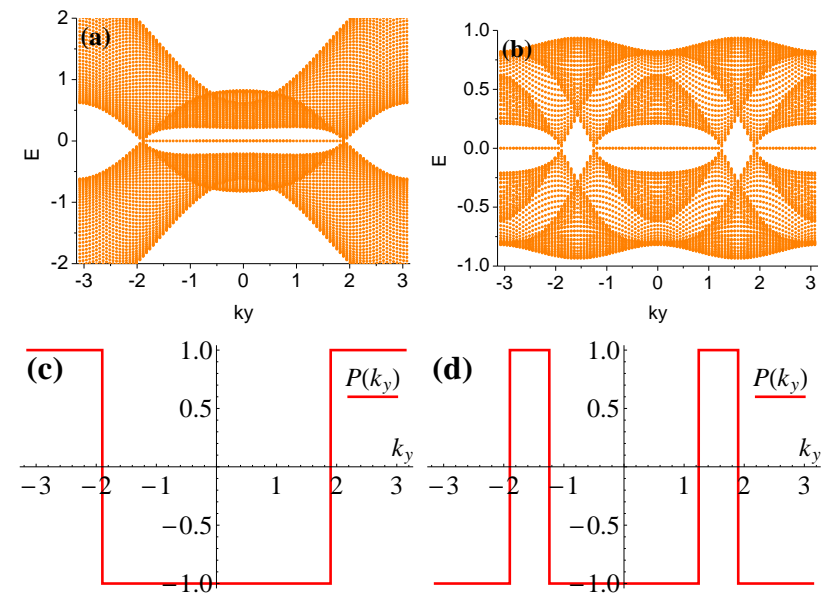

FIG. 3. (color online). (a) and (b) are the edge spectra of the $s$-wave superconductor with Dresselhaus (110) spin-orbit coupling in case (c) of Tab. (II). The parameters are $t=1$, $\beta=1, \Delta_{s_{1}}=1, \Delta_{s_{2}}=1$ and (a) $\mu=-4, V^{2}=9$, (b) $\mu=0, V^{2}=9$, which correspond to regions I and II in Fig. (17), respectively. (c) and (d) are the Pfaffian invariant for (a) and (b).

\section{SUMMARY}

In summary, we have investigated the topological phase and the Majorana bound state in the spin-singlet superconductor with the Rashba and Dresselhaus (110) spin-orbit couplings. We find that apart from the 

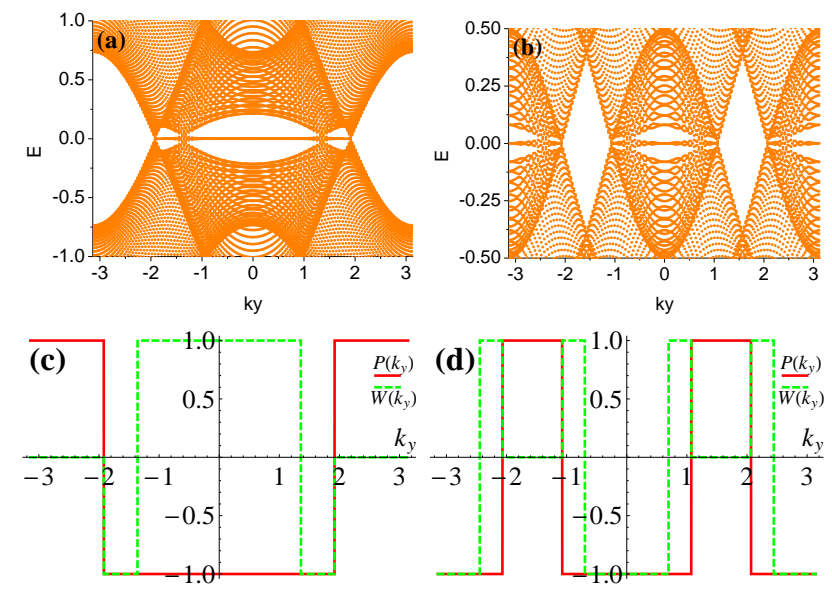

FIG. 4. (color online). (a) and (b) are the edge spectra of the $d_{x^{2}-y^{2}}$-wave superconductor with Dresselhaus (110) spinorbit coupling in case (d) of Tab. (I). The parameters are $t=1, \beta=1, \Delta_{d_{1}}=1, \Delta_{d_{2}}=0$ and (a) $\mu=-4, V^{2}=9$, (b) $\mu=0, V^{2}=9$, which correspond to regions I and II in Fig. (1a), respectively. (c) and (d) are the Pfaffian invariant and winding number for (a) and (b).
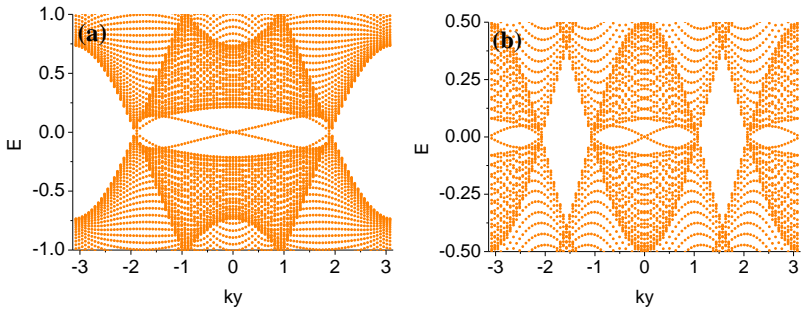

FIG. 5. (color online). (a) and (b) are the edge spectra of the $d_{x^{2}-y^{2}}+i d_{x y}$-wave superconductor with Dresselhaus (110) spin-orbit coupling in case (e) of Tab. (I). The parameters are $t=1, \beta=1, \Delta_{d_{1}}=1, \Delta_{d_{2}}=1$ and (a) $\mu=-4, V^{2}=9$, (b) $\mu=0, V^{2}=9$, which correspond to regions I and II in Fig. (1), respectively. particle-hole symmetry, the BdG Hamiltonian can also exhibit the chiral symmetry, partial particle-hole symmetry and partial chiral symmetry as listed in Tab. (I). The topological invariants corresponding to these symmetries have been discussed in Sec. (IV) and are also listed in Tab. (I). We have demonstrated all the possible phase diagrams of the spin-singlet superconductor with the Rashba and Dresselhaus (110) spin-orbit couplings in Sec. (V) by the Pfaffian invariant $\mathcal{P}$ of the particlehole symmetric Hamiltonian. We find that only when the Hamiltonian has partial particle-hole symmetry or chiral symmetry, the edge spectrum is flat band protected by the one dimensional Pfaffian invariant $\mathcal{P}\left(k_{y}\right)$ or the winding number $\mathcal{W}\left(k_{y}\right)$; otherwise the edge spectrum is Dirac cone. The zero-energy state of the Dirac cones and the zero-energy flat bands are the Majorana type which is a precious source for topological quantum computing. The edge spectra of the cases listed in Tab. (II) are shown in Sec. (VI). We find that the Pfaffian invariant $\mathcal{P}\left(k_{y}\right)$ and the winding number $\mathcal{W}\left(k_{y}\right)$ can be used in determining the location of the zero-energy flat bands.

\section{ACKNOWLEDGMENTS}

This work is partly supported by the National Research Foundation and Ministry of Education, Singapore (Grant No. WBS: R-710-000-008-271).
* jiabinyou@gmail.com

$\dagger$ phycahp@nus.edu.sg

phyohch@nus.edu.sg

$\S$ phyvv@nus.edu.sg

1 N. Read and D. Green, Phys. Rev. B 61, 10267 (2000)

2 L. Fu and C. L. Kane, Phys. Rev. Lett. 100, 096407 (2008).

${ }^{3}$ M. Sato, Y. Takahashi, and S. Fujimoto, Phys. Rev. B 82, $134521(2010)$

${ }^{4}$ J. Alicea, Phys. Rev. B 81, 125318 (2010)

5 Y. Oreg, G. Refael, and F. von Oppen, Phys. Rev. Lett. 105, $177002(2010)$

${ }^{\circ}$ T. H. Hsieh and L. Fu, Phys. Rev. Lett. 108, 107005 (2012)

S. Tewari and J. D. Sau, Phys. Rev. Lett. 109, 150408 (2012)

$\checkmark$ J. You, C. H. Oh, and V. Vedral, Phys. Rev. B 87, 054501 (2013)
9 J. D. Sau, R. M. Lutchyn, S. Tewari, and S. Das Sarma, Phys. Rev. Lett. 104, 040502 (2010)

10 M. Sato and S. Fujimoto, Phys. Rev. Lett. 105, 217001 (2010)

11 A. P. Schnyder and S. Ryu, Phys. Rev. B 84, 060504 (2011)

12 M. Lababidi and E. Zhao, Phys. Rev. B 86, 161108 (2012)

13 M. Sato, Y. Tanaka, K. Yada, and T. Yokoyama, Phys. Rev. B 83, 224511 (2011).

${ }^{14}$ P. M. R. Brydon, A. P. Schnyder, and C. Timm, Phys. Rev. B 84, 020501 (2011).

To C. L. M. Wong, J. Liu, K. T. Law, and P. A. Lee, arXiv:1206.5601v3.

${ }^{16}$ K. Nakada, M. Fujita, G. Dresselhaus, and M. S. Dresselhaus, Phys. Rev. B 54, 17954 (1996).

17 G. Volovik, JETP Letters 93, 66 (2011)

18 Y. Tanaka, Y. Mizuno, T. Yokoyama, K. Yada, and M. Sato, Phys. Rev. Lett. 105, 097002 (2010) 

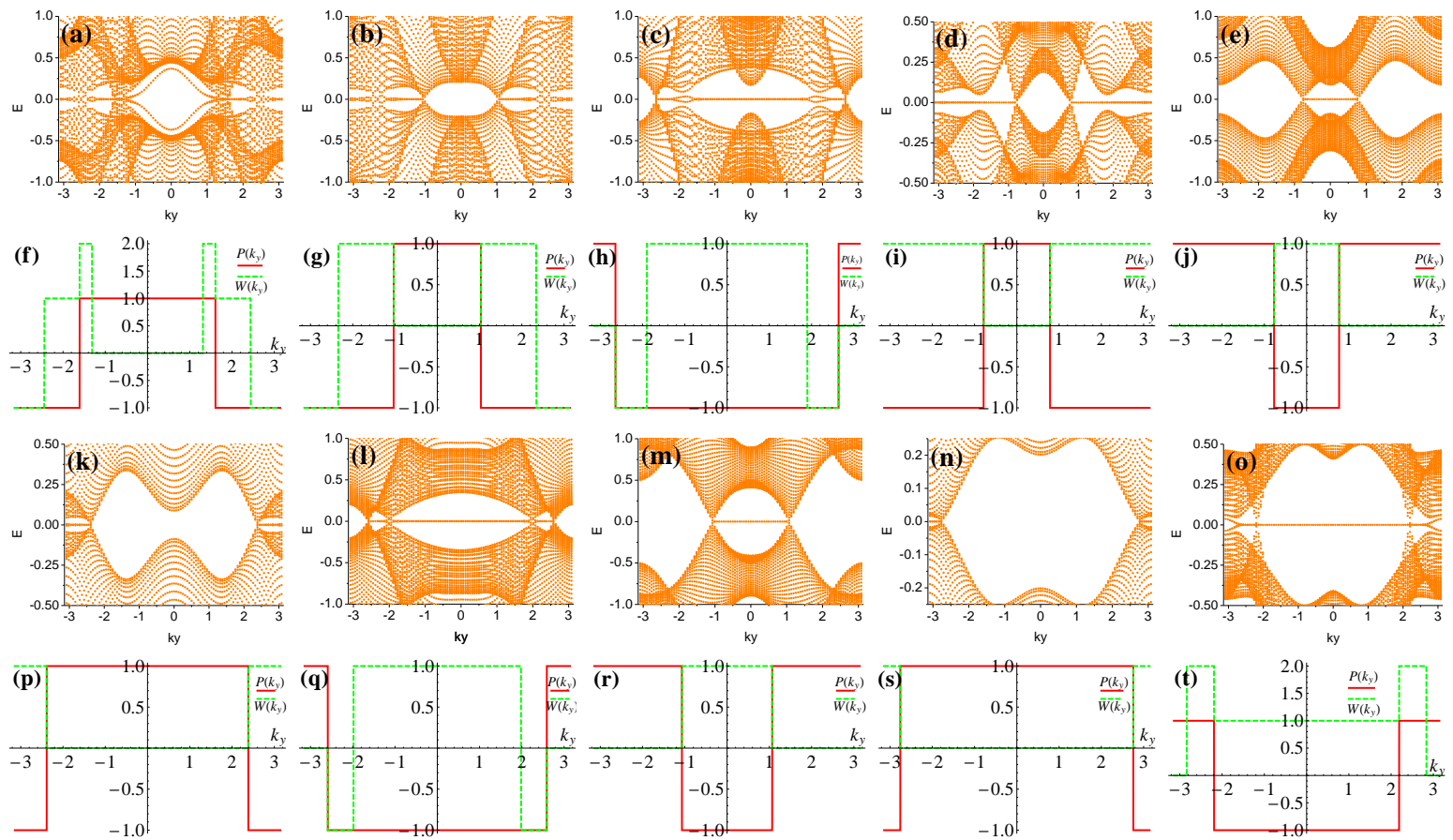

FIG. 6. (color online). (a)-(e) and (k)-(o) are the edge spectra of the $d_{x^{2}-y^{2}}+s$-wave superconductor with Dresselhaus (110) spin-orbit coupling in case (f) of Tab. (I). There are three different kinds of phase diagrams depending on the hopping amplitude $t$ as shown in Fig. $(1 \mathrm{~b})-(1 \mathrm{~d})$. For the phase diagram of Fig. $(1 \mathrm{~b})$, the edge spectra are demonstrated in (a), (b) and (c). The parameters are $t=2, \beta=1, \Delta_{s_{1}}=1, \Delta_{d_{1}}=2$ and (a) $\mu=0, V^{2}=16$, (b) $\mu=-2.5, V^{2}=36,(\mathrm{c}) \mu=-4, V^{2}=20$, which correspond to regions I, II and III in Fig. (1p), respectively. (f), (g) and (h) are the Pfaffian invariant and winding number for (a), (b) and (c). For the phase diagram of Fig. (1k), the edge spectra are demonstrated in (d), (e), (k) and (l). The parameters are $t=1, \beta=1, \Delta_{s_{1}}=1, \Delta_{d_{1}}=2$ and (d) $\mu=0, V^{2}=12,(\mathrm{e}) \mu=0, V^{2}=20$, (k) $\mu=-1.8, V^{2}=30,(\mathrm{l}) \mu=-4.5, V^{2}=25$, which correspond to regions I, II, III and IV in Fig. (1), respectively. (i), (j), (p) and (q) are the Pfaffian invariant and winding number for (d), (e), (k) and (l). For the phase diagram of Fig. (1 $\mathrm{d})$, the edge spectra are demonstrated in (m), (n) and (o). The parameters are $t=0.5, \beta=1, \Delta_{s_{1}}=1, \Delta_{d_{1}}=2$ and $(\mathrm{m}) \mu=0, V^{2}=16$, (n) $\mu=-7, V^{2}=81$, (o) $\mu=-1, V^{2}=5$, which correspond to regions I, II and III in Fig. (1) , respectively. (r), (s) and (t) are the Pfaffian invariant and winding number for $(\mathrm{m}),(\mathrm{n})$ and $(\mathrm{o})$.
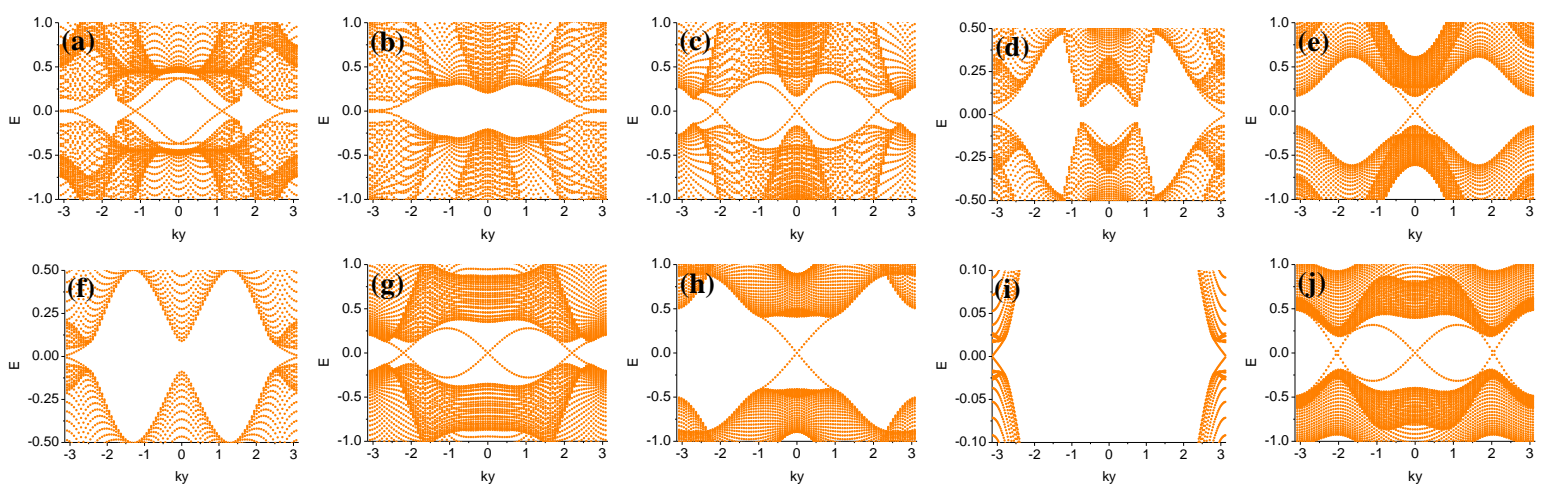

FIG. 7. (color online). (a)-(j) are the edge spectra of the $d_{x^{2}-y^{2}}+i d_{x y}+s$-wave superconductor with Rashba spin-orbit coupling in case (a) of Tab. (I). For the phase diagram of Fig. (1p), the edge spectra are demonstrated in (a), (b) and (c). The parameters are $t=2, \alpha=1, \Delta_{s_{1}}=1, \Delta_{s_{2}}=0, \Delta_{d_{1}}=2, \Delta_{d_{2}}=1$ and (a) $\mu=0, V_{z}=4$, (b) $\mu=-2.5, V_{z}=6,(\mathrm{c})$ $\mu=-4, V_{z}=2 \sqrt{5}$, which correspond to regions I, II and III in Fig. (1 b), respectively. For the phase diagram of Fig. (1 $\mathrm{k}$ ), the edge spectra are demonstrated in (d), (e), (f) and (g). The parameters are $t=1, \alpha=1, \Delta_{s_{1}}=1, \Delta_{s_{2}}=0, \Delta_{d_{1}}=2, \Delta_{d_{2}}=1$ and (d) $\mu=0, V_{z}=2 \sqrt{3}$, (e) $\mu=0, V_{z}=2 \sqrt{5}$, (f) $\mu=-1.8, V_{z}=\sqrt{30}$, (g) $\mu=-4.5, V_{z}=5$, which correspond to regions I, II, III and IV in Fig. (1F), respectively. For the phase diagram of Fig. (1/ d), the edge spectra are demonstrated in (h), (i) and (j). The parameters are $t=0.5, \alpha=1, \Delta_{s_{1}}=1, \Delta_{s_{2}}=0, \Delta_{d_{1}}=2, \Delta_{d_{2}}=1$ and (h) $\mu=0, V_{z}=4,(\mathrm{i}) \mu=-7, V_{z}=9,(\mathrm{j})$ $\mu=-3, V_{z}=3$, which correspond to regions I, II and III in Fig. (1), respectively. 

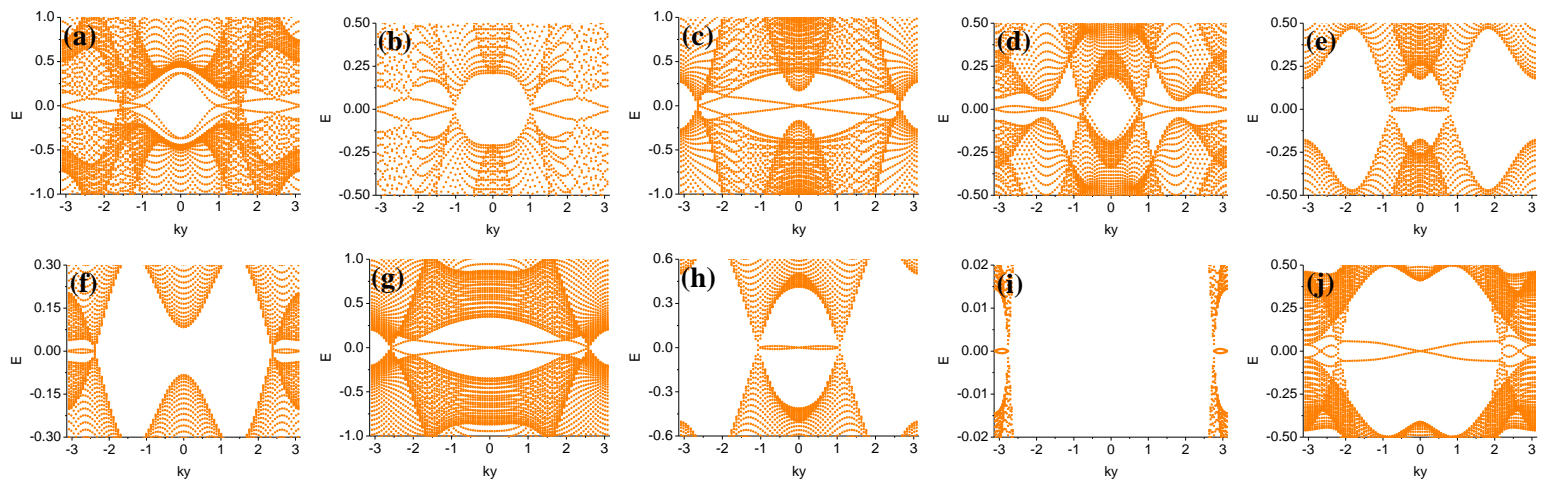

FIG. 8. (color online). (a)-(j) are the edge spectra of the $d_{x^{2}-y^{2}}+i d_{x y}+s$-wave superconductor with Dresselhaus (110) spin-orbit coupling in case (g) of Tab. (I). For the phase diagram of Fig. (1p), the edge spectra are demonstrated in (a), (b) and (c). The parameters are $t=2, \beta=1, \Delta_{s_{1}}=1, \Delta_{s_{2}}=0, \Delta_{d_{1}}=2, \Delta_{d_{2}}=1$ and (a) $\mu=0, V^{2}=16,(\mathrm{~b}) \mu=-2.5, V^{2}=36$, (c) $\mu=-4, V^{2}=20$, which correspond to regions I, II and III in Fig. (1p), respectively. For the phase diagram of Fig. (1p), the edge spectra are demonstrated in (d), (e), (f) and (g). The parameters are $t=1, \beta=1, \Delta_{s_{1}}=1, \Delta_{s_{2}}=0, \Delta_{d_{1}}=2, \Delta_{d_{2}}=1$ and (d) $\mu=0, V^{2}=12$, (e) $\mu=0, V^{2}=20$, (f) $\mu=-1.8, V^{2}=30$, (g) $\mu=-4.5, V^{2}=25$, which correspond to regions I, II, III and IV in Fig. (1F), respectively. For the phase diagram of Fig. (1) , the edge spectra are demonstrated in (h), (i) and (j). The parameters are $t=0.5, \beta=1, \Delta_{s_{1}}=1, \Delta_{s_{2}}=0, \Delta_{d_{1}}=2, \Delta_{d_{2}}=1$ and $(\mathrm{h}) \mu=0, V^{2}=16,(\mathrm{i}) \mu=-7, V^{2}=81,(\mathrm{j})$ $\mu=-1, V^{2}=5$, which correspond to regions I, II and III in Fig. (1), respectively.

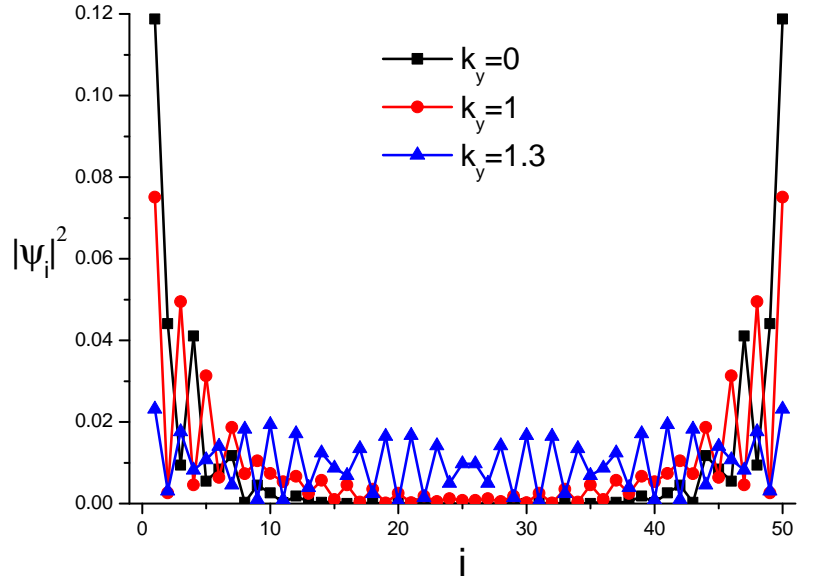

FIG. 9. (color online). The probability distributions of the quasiparticle in the $d_{x^{2}-y^{2}}$-wave superconductor with Dresselhaus (110) spin-orbit coupling in the edge Brillouin zone of $k_{y}=0,1,1.3 . \quad i$ is the lattice site. $\left|\psi_{i}\right|^{2}$ is the probability of quasiparticle at site $i$.
19 V. Mourik, K. Zuo, S. M. Frolov, S. R. Plissard, E. P. A. M. Bakkers, and L. P. Kouwenhoven, Science 336, 1003 (2012).

20 A. Das, Y. Ronen, Y. Most, Y. Oreg, M. Heiblum, and H. Shtrikman, Nat. Phys. 8, 887 (2012).

21 S. Sasaki, M. Kriener, K. Segawa, K. Yada, Y. Tanaka, M. Sato, and Y. Ando, Phys. Rev. Lett. 107, 217001 (2011)

22 T. V. Bay, T. Naka, Y. K. Huang, H. Luigjes, M. S. Golden, and A. de Visser, Phys. Rev. Lett. 108, 057001 (2012)

23 A. Kitaev, Phys. Usp. 44, 131 (2001).

${ }^{24}$ C. Nayak, S. H. Simon, A. Stern, M. Freedman, and S. Das Sarma, Rev. Mod. Phys. 80, 1083 (2008)

25 R. Winkler, Spin-Orbit Coupling Effects in TwoDimensional Electron and Hole Systems (Springer-Verlag Berlin Heidelberg, 2003).

${ }^{26}$ P. Ghosh, J. D. Sau, S. Tewari, and S. Das Sarma, Phys. Rev. B 82, 184525 (2010). 